\title{
Pressure from particle image velocimetry for convective flows: A Taylor's hypothesis approach
}

\author{
Roeland de Kat ${ }^{1}$ and Bharathram Ganapathisubramani \\ Aerodynamics and Flight Mechanics Research Group, University of Southampton, UK \\ 1 r.de-kat@soton.ac.uk
}

\begin{abstract}
Taylors hypothesis is often applied in turbulent flow analysis to map temporal information into spatial information. Recent efforts in deriving pressure from particle image velocimetry (PIV) have proposed multiple approaches, each with its own weakness and strength. Application of Taylors hypothesis allows us to counter the weakness of an Eulerian approach that is described by de Kat \& van Oudheusden (2012 Exp. Fluids 52 1089106). We build on the findings of de Kat \& Ganapathisubramani (2013 Meas. Sci. Technol. 24 024002) and look in more detail into different ways of obtaining estimates for convection velocity on the determination of pressure from PIV using Taylor's hypothesis. We also look at the influence of the omission of viscous terms. Results appear to indicate that pressure can indeed be obtained from PIV data in turbulent convective flows using the Taylors hypothesis approach, where there are no other methods to determine pressure. A more local estimate of convection velocity results in a pressure field that has less obvious defects. Other than a change in reference pressure for the pressure evaluation, inclusion or omission of the viscous terms appears to not have a significant effect.
\end{abstract}

\section{Introduction}

Interest for deriving instantaneous pressure from particle image velocimetry (PIV) is increasing. Several studies have covered a variety of approaches to accomplish this. Liu \& Katz [1] apply four-exposure PIV together with a Lagrangian approach to determine the material acceleration and pressure fields. Charonko et al. [2] investigate various Eulerian approaches and showed results for a diverging channel. Violato et al. [3] apply Lagrangian and Eulerian approaches to a rod-airfoil configuration. De Kat \& van Oudheusden [5] test Lagrangian and Eulerian approaches on synthetic data and compared pressure from PIV with pressure transducer signals in a square cylinder flow. Van Oudheusden [4] gives a comprehensive review of different PIV-based techniques to obtain pressure.

For turbulent convective flows there are some issues that trouble the determination of pressure from PIV. Time-resolved volumetric PIV (TR-Volume) measure everything needed to determine the pressure gradient. However, Eulerian approaches, due to their sensitivity to measurement noise in combination with convection, are not very well suited to capture convective flow [5] using TR-Volume data. Lagrangian approaches, while less sensitive to measurement noise, are hampered by the need for measurement volumes of sufficient size to reconstruct fluid/particle trajectories [5] and, therefore, sacrifice spatial resolution or domain size (the number of particles that can be captured by sensors is limited) in favour of trajectory reconstruction.

Taylor's hypothesis is often applied in turbulent flow analysis to map temporal information into spatial information using a convection velocity. We use Taylor's hypothesis in combination with an Eulerian method to address the limitations of obtaining pressure from PIV for convective flows.

In this paper, we summarise the findings of de Kat \& Ganapathisubramani [6] and look in more detail into different ways of obtaining estimates for convection velocity on the determination of pressure from PIV using Taylor's hypothesis. We also look at the influence of the omission of viscous terms. De Kat \& Ganapathisubramani [6] address the limitations of obtaining pressure from PIV for convective flows by using Taylor's hypothesis in combination with an Eulerian method. First, the methodology that is used to derive pressure from PIV is introduced and the uncertainties associated with the method are considered. Last, the method is applied to experimental data obtained in a crossflow plane in the far-field of a turbulent jet [7,8]. This data set is used to investigate different estimates for convection velocity and the influence of viscosity.

\section{Pressure determination from PIV using Taylor's hypothesis}

The determination of pressure in a plane that is used in this work is described in detail by de Kat \& van Oudheusden [5]. In short, the momentum equation is rewritten to obtain the pressure gradient:

$$
\nabla p=-\rho\left\{\frac{\partial \mathbf{u}}{\partial t}+(\mathbf{u} \cdot \nabla) \mathbf{u}-v \nabla^{2} \mathbf{u}\right\}
$$

For fully developed convective turbulent flow (high Reynolds numbers) the acceleration and convective terms dominate and the viscous term can be neglected. The terms in the pressure gradients are evaluated with central differences (Eulerian approach). The pressure 

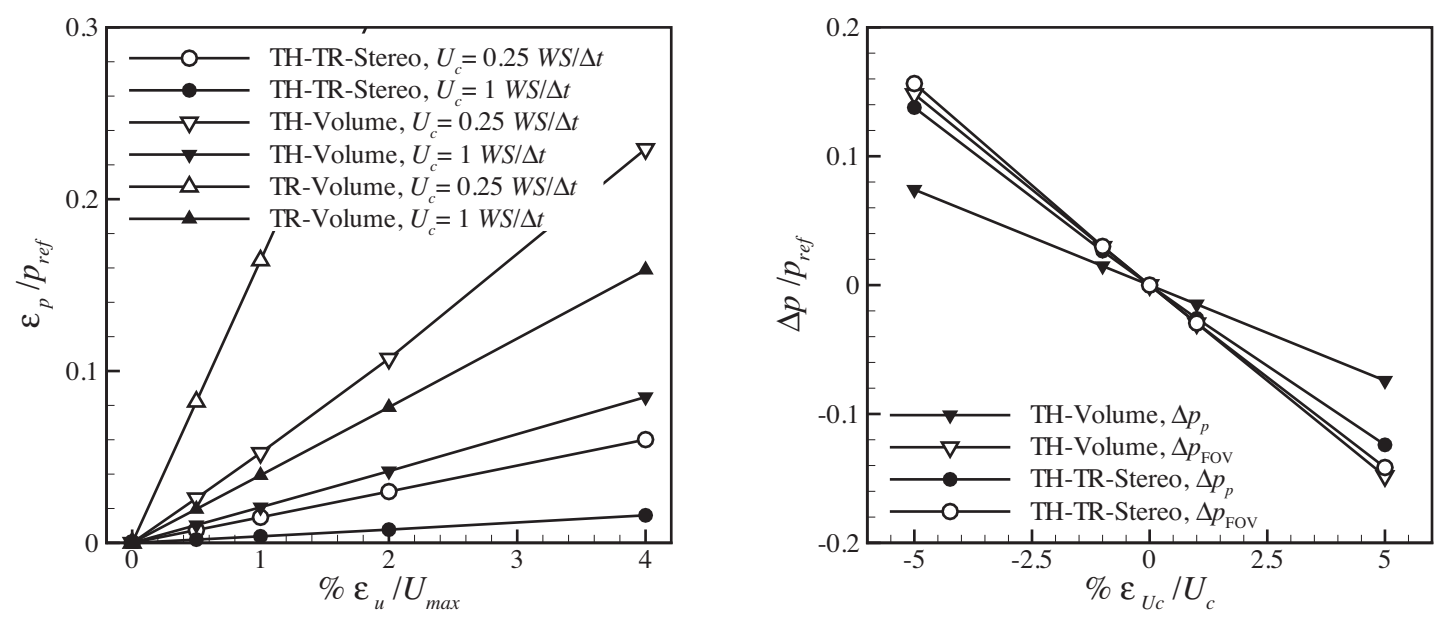

Figure 1: Influence of noise and accuracy of convection velocity on pressure determination results [6]. left Random error in pressure with random error in velocity for different techniques. right Error in peak pressure and asymmetry due to error in convection velocity.

gradient is spatially integrated using a Poisson formulation, which involves taking the in-plane divergence of the pressure gradient and subsequently integrating that result with a Poisson solver.

$$
\nabla_{y z} \cdot \nabla p=\frac{\partial^{2} p}{\partial y^{2}}+\frac{\partial^{2} p}{\partial z^{2}}=\left(\frac{\partial}{\partial y}\left(\left.\frac{\partial p}{\partial y}\right|_{\mathrm{PIV}}\right)+\frac{\partial}{\partial z}\left(\left.\frac{\partial p}{\partial z}\right|_{\mathrm{PIV}}\right)\right)
$$

The in-plane divergence of a vector function, $\mathbf{g}$, is $\nabla_{y z} \cdot \mathbf{g}=\partial g_{y} / \partial y+\partial g_{z} / \partial z$, where $g_{y}$ and $g_{z}$ are the components in $y$-direction and $z$-direction respectively.

De Kat \& van Oudheusden [5] show that with TR-Volume measurements (in their case the volumetric method was tomographic-PIV, see [9]) good results can be obtained when the flow is dominated by strong vortices that do not convect at large velocities. They found that for convective flows with relatively weak vortices (small induced velocity with respect to the convection velocity) an Eulerian approach will struggle.

In order to overcome the difficulties encountered by the Eulerian approach in convective flows, de Kat \& Ganapathisubramani [6] propose a new method where either crossflow plane time-resolved PIV data or tomographic PIV data (not time-resolved) can be used in liaison with Taylor's hypothesis to calculate planar or volumetric pressure fields.

The velocity, $\mathbf{u}$ (with $u$ as streamwise velocity component and $v$ and $w$ as cross-flow velocity components), is decomposed into its mean, $\mathbf{U}$, and its fluctuation around the temporal mean, $\mathbf{u}^{\prime}$. A fluctuation (or perturbation) is convected by the flow at a certain convection velocity, $\mathbf{U}_{\mathbf{c}}$. If the fluctuation is 'frozen', the temporal and spatial gradients are linked by the convection velocity.

When this relation is substituted in equation 1, de Kat \& Ganapathisubramani [6] show that

$$
\nabla p_{\mathrm{TH}}=-\rho\left\{\left(\mathbf{u}^{\prime} \cdot \nabla\right) \mathbf{u}+\left(\left[\mathbf{U}-\mathbf{U}_{\mathbf{c}}\right] \cdot \nabla\right) \mathbf{u}^{\prime}\right\}
$$

As can be seen from equation 3, the determination of the pressure gradient can be done without any temporal information, provided that the convection velocity is known. This would mean that Taylor's hypothesis combined with instantaneous volumetric velocity measurement (TH-Volume, where the volumetric measurement can be tomographic-PIV, holographic-PIV, or scanning-PIV) would suffice to determine the pressure gradient, which can then be integrated to get volumetric or planar pressure, provided the mean velocity and convection velocity are known.

In most convective flows (e.g.boundary layers, jets, channels, etc.), there is one dominant direction of flow. For these flows, $V$ and $W$ are negligible compared to $U$, the mean velocity in streamwise direction. Therefore, we expect the convection velocity to follow a similar trend (i.e. $V_{c}=W_{c} \approx 0$ ). In this case, a different approach can be used: Taylor's hypothesis combined with time-resolved stereoscopic-PIV in a crossflow plane (TH-TR-Stereo), where Taylor's hypothesis is also used to map the temporal information into space with

$$
\frac{\partial \mathbf{u}^{\prime}}{\partial x}=-\frac{1}{U_{c}} \frac{\partial \mathbf{u}^{\prime}}{\partial t}
$$

and as such to obtain the out-of-plane ( $x$-direction) velocity gradients. In this way, TH-TR-Stereo can be used to determine the pressure in the crossflow plane ( $y-z$ plane). In some cases, scanning or dual-plane PIV can also be employed.

The main advantage of using Taylor's hypothesis is that it is less sensitive to errors in the velocity field than time-resolved techniques. Assessment of performance of the approach on synthetic data shows that using a TH-TR-Stereo approach would introduce the smallest 

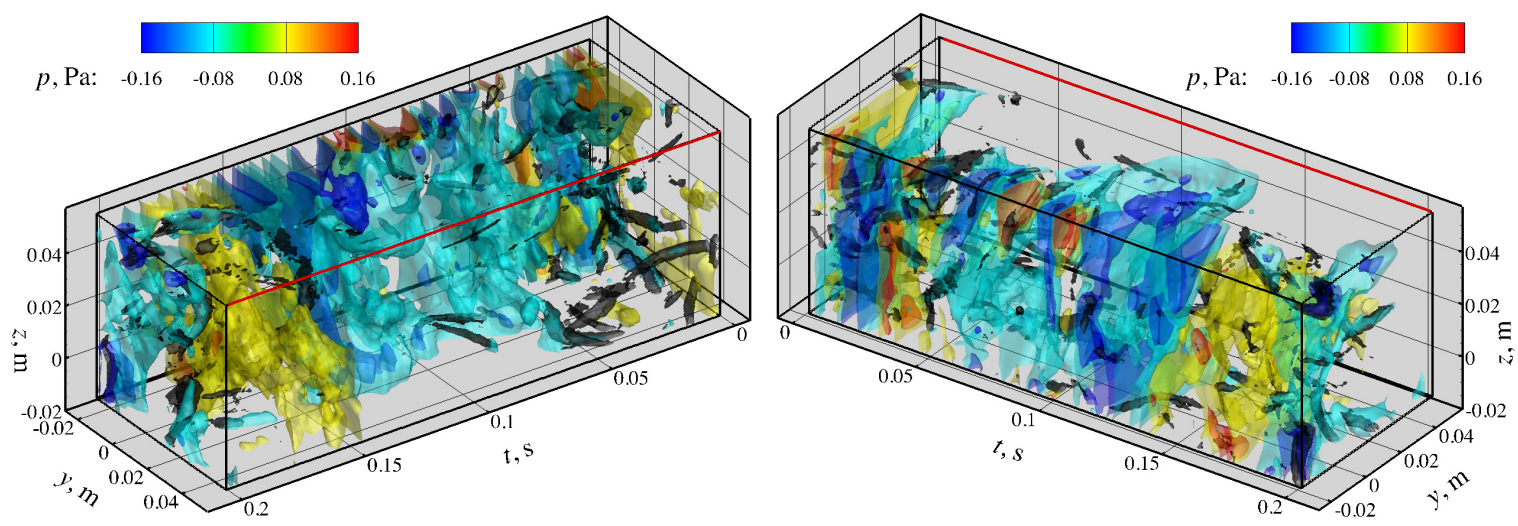

Figure 2: Two views of the space-time volume of pressure with $U_{c}=U$ (view on the left from [6]). The location of the reference pressure is depicted by the red edge. Isocontours of vorticity magnitude $\left(|\omega|=200 \mathrm{~s}^{-1}\right)$ are shown in black. Isosurfaces of pressure are shown for $-0.16 \mathrm{~Pa},-0.08 \mathrm{~Pa}, 0.08 \mathrm{~Pa}$, and $0.16 \mathrm{~Pa}$.

error in the pressure field for the same error in the velocity field compared to TR-Volume and TH-Volume, see figure 1left. However, this requires the convection velocity to be known. The sensitivity of the TH-approaches shows that a mismatch in convection velocity shows up as an error in the peak pressure determined and an asymmetry in the pressure field, caused by in-plane vortices moving through the measurement plane, see figure 1right.

The success of $\mathrm{TH}$-approaches relies on being able to determine a (reasonable) estimate for the convection velocity of the structures passing through the measurement plane. If the estimate is off, strong (unrealistic) pressure fluctuations will be expected on the opposite side of the reference pressure. Application of the TH-TR-Stereo approach to the far-field of a turbulent jet will demonstrate this.

\section{Pressure fluctuations in a turbulent jet}

To illustrate the capabilities of this technique, de Kat \& Ganapthisubramani [6] applied this technique to the far-field of a turbulent jet, as measured by [7], who applied cinematographic stereo-PIV (i.e. time-resolved stereo-PIV).

\subsection{Mean velocity as convection velocity}

Using the TH-TR-Stereo approach, pressure is determined using the mean velocity as the convection velocity, $U_{c}=U$. This estimate for convection velocity varies in the crossflow direction ranging from approximately $0.4 \mathrm{~m} / \mathrm{s}$ to $0.8 \mathrm{~m} / \mathrm{s}$. For this experiment, the spatial resolution with respect to the typical vortices in the flow is $0.6 \mathrm{WS} / r_{v}$, where $r_{v}$ is the radius of a typical vortex. The convection velocity is range is $U_{c} \approx 0.3-0.6 \mathrm{WS} / \Delta t=0.2-0.3 r_{v} / \Delta t$, and the tangential velocity can be estimated as three times the turbulence intensity, $V_{p} / U_{c} \approx 0.9$. Based on the synthetic data analysis these values should give good pressure results, provided that the mean velocity is a good estimate for the convection velocity.

The space-time pressure volume in figure 2 shows strong oscillations on the far side(s) of the reference pressure. They are clearly visible along the top edge opposite from the reference point. The oscillations are related to in-plane vortices passing through the measurement plane and can be explained as asymmetries that are introduced by using an incorrect convection velocity [6].

\subsection{Local averages as convection velocity}

In order to try to overcome the problem that we observed with using the mean velocity as convection velocity, three different ways of estimating the convection velocity is to used. Elsinga et al. [10] found that the difference between the local flow velocity and the convection velocity is smaller than the difference between the mean velocity and the convection velocity by tracking hairpin structures in a turbulent boundary layer. Based on this observation, convection velocities can be estimated by local averages of the streamwise velocity. First, the instantaneous axial velocity is filtered in-plane by a moving average over a kernel with sides $l_{f}$ to obtain the estimate for the convection velocity, $[u]_{l_{f}}[6]$. Second, the instantaneous axial velocity is filtered in time by a moving average over a kernel with length $t_{f}$ to obtain the estimate for the convection velocity, $[u]_{t_{f}}$. Finally, the instantaneous axial velocity is filtered in-plane and in time by a moving average over a kernel with sides $l_{f}$ in space and length $t_{f}$ in time to obtain the estimate for the convection velocity, $[u]_{l_{f}, t_{f}}$. To examine how different filter sizes affect the input to the pressure solver, the relative change of the divergence of the velocity field and the relative change of the curl of the in-plane pressure gradient with respect to the results obtained from with the mean velocity as a convection velocity estimate are determined. Ideally, both quantities should have values that are zero, however, due to measurement errors they will have a certain spread. Therefore, the bigger the decrease in relative change the better the estimate for convection velocity. 

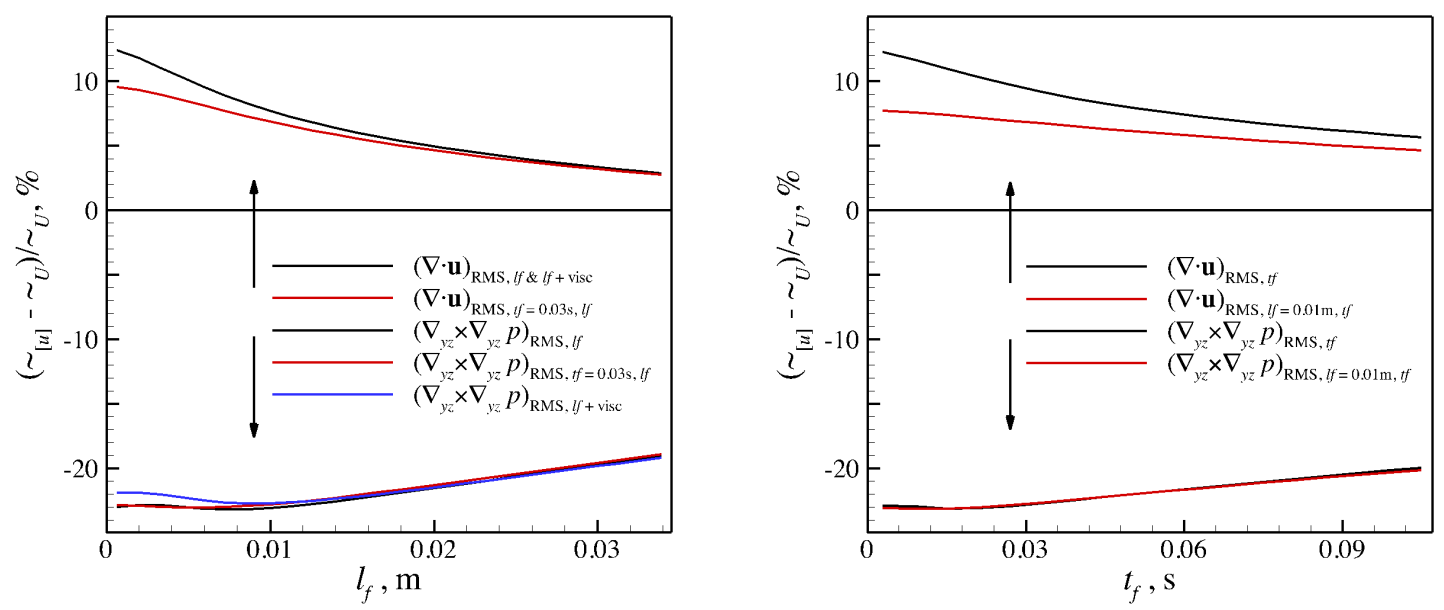

Figure 3: Filter length and type influence. left Influence of spatial filter length on $\nabla \cdot \mathbf{u}$ and $\nabla_{y z} \times \nabla_{y z} p$. The space-time filter has $t_{f}=0.03 \mathrm{~s}$. right Influence of temporal filter length on $\nabla \cdot \mathbf{u}$ and $\nabla_{y z} \times \nabla_{y z} p$. The space-time filter has $l_{f}=0.1 \mathrm{~m}$.

Figure 3 shows the result of this analysis. For the spatial filtered estimate for convection velocity (3left, black lines), we can see a positive change in divergence indicating that there is less agreement in terms of continuity. This amount of change decreases with increasing $l_{f}$. Even though the result is worse in terms of continuity, the change in curl of the in-plane pressure gradient is negative, which means the resulting pressure gradient is better. The error in curl is $23 \%$ lower up to $l_{f}=10 \mathrm{~mm}$. Near the smallest value (smallest value means no filter, which is equal to a $2 \mathrm{D}$ approach) the change is slightly smaller.

To obtain the largest improvement for the curl with the smallest deterioration in the divergence, $l_{f}=10 \mathrm{~mm}$ is used, which corresponds to twice the typical vortex tube diameter [8]. Even though this approach to estimate convection velocity deteriorates continuity, the reduction in error for the pressure gradient is evident. As the goal is to get a better estimate for the pressure field, this deterioration of continuity is of less importance than the improvement of the pressure gradient input.

For the temporal filtered estimate for convection velocity (3right, black lines), we can also see a positive change in divergence indicating that there is less agreement in terms of continuity. This amount of change decreases with increasing $t_{f}$. Again, even though the result is worse in terms of continuity, the change in curl of the in-plane pressure gradient is negative, which means the resulting pressure gradient is better. The error in curl is $23 \%$ lower up to $t_{f}=30 \mathrm{~ms}$. Near the smallest value (smallest value means no filter, which is equal to a $2 \mathrm{D}$ approach) the change is slightly smaller. For this convection velocity, the spatial resolution and the estimated peak tangential velocity remain the same, however the convection velocities now range from $U_{c} \approx 0.1-0.9 \mathrm{WS} / \Delta t=0.05-0.5 r_{v} / \Delta t$. This values should still give good pressure results based on the synthetic data analysis, provided the new estimates for convection velocity are better.

Figure 4top shows the resulting space-time pressure volume. The strong oscillations have disappeared and collocated low pressure and high vorticity can be seen in the top of the volume, whereas in 2 there was no clear link between the vorticity and pressure. The volume shows large regions of high and low pressure with strong vortices piercing through these regions creating locally lower pressure.

To obtain the largest improvement for the curl with the smallest deterioration in the divergence, $t_{f}=30 \mathrm{~ms}$ is used. Even though this approach to estimate convection velocity deteriorates continuity, the reduction in error for the pressure gradient is evident. As the goal is to get a better estimate for the pressure field, this deterioration of continuity is of less importance than the improvement of the pressure gradient input. Figure 4middle shows the resulting space-time pressure volume. Again, compared with the results for the mean velocity as convection velocity, the strong oscillations have disappeared and collocated low pressure and high vorticity can be seen in the top of the volume. Comparing this result with the result for the space filter (4top), we see that both the time filtered and the space filtered estimates for convection velocity result in similar pressure volumes, which one would expect based on the similar improvement in curl of the in-plane pressure gradient.

Filtering in both space and time does not result in a better result for the curl of the in-plane pressure gradient. A spatial filter length sweep with a constant time filter (3left, red lines) shows that there is a small decrease in divergence. A temporal filter length sweep with a constant space filter (3right, red lines) shows that there is a more significant decrease in divergence. If we look at the 'optimal' filter lengths for reducing the curl in the in-plane pressure gradient and compare the space-time filtered results (red lines) to the space or time filtered results (black lines), we see that filtering only in space is more beneficial for the divergence than filtering only in time. Figure 4 bottom shows the space-time pressure volume for $t_{f}=30 \mathrm{~ms}$ and $l_{f}=10 \mathrm{~mm}$. As before, the pressure volume is similar to the results of both the time filtered and the space filtered estimates for convection velocity.

\subsection{Influence of viscous terms}

Most works that use PIV-based pressure determination approaches, neglect the viscous terms. However, this is not necessarily a valid 

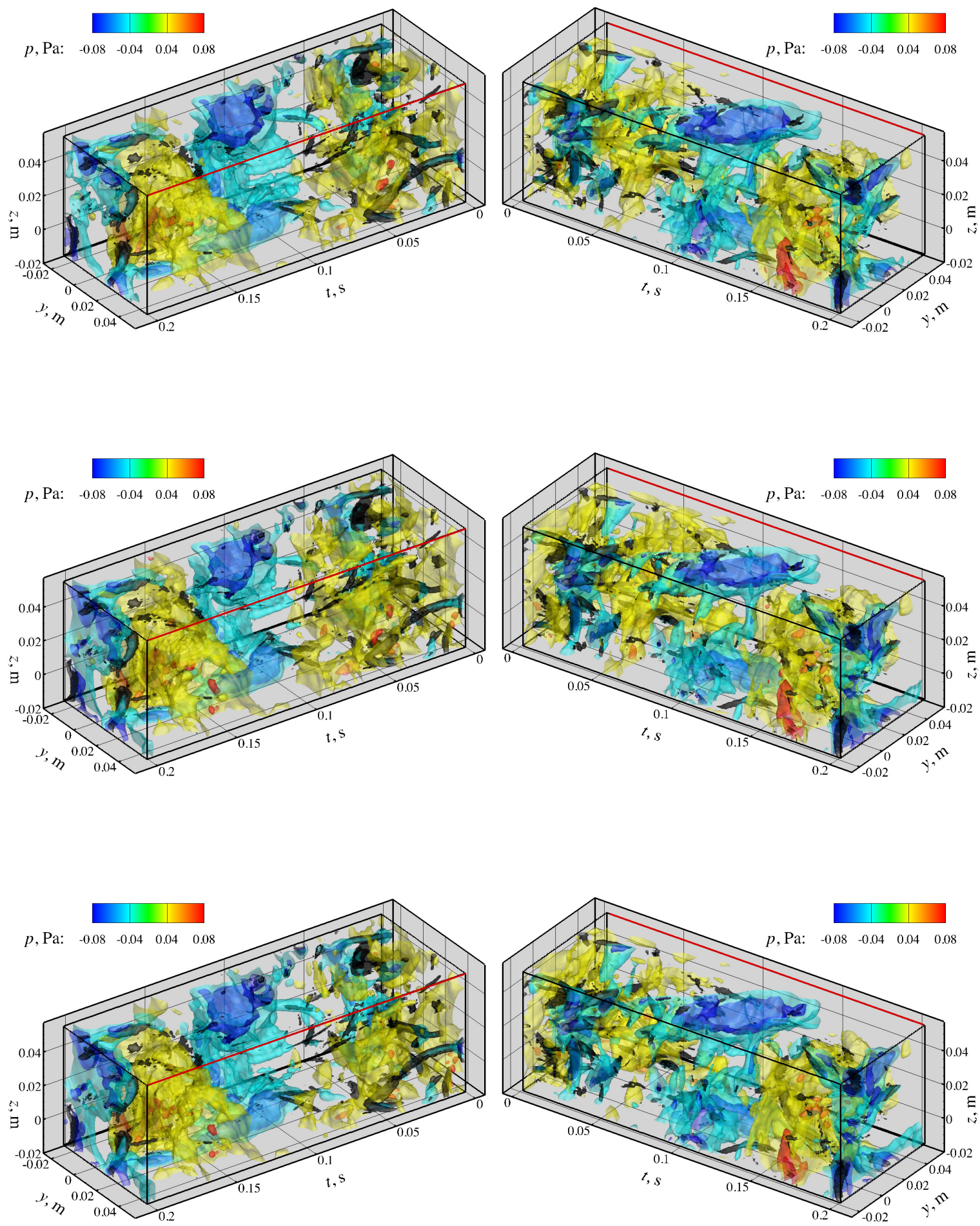

Figure 4: Views of space-time volume of pressure for different convection velocity estimates. The location of the reference pressure is depicted by the red edge. Isocontours of vorticity magnitude $\left(|\omega|=200 \mathrm{~s}^{-1}\right)$ are shown in black. Isosurfaces of pressure are shown for -0.08 Pa, - $0.04 \mathrm{~Pa}, 0.04 \mathrm{~Pa}$, and $0.08 \mathrm{~Pa}$. Top Two views of space-time pressure for $U_{c}=[u]_{l_{f}}$. (view on the left from [6]). Middle Two views of space-time pressure for $U_{c}=[u]_{t_{f}}$. Bottom Two views of space-time pressure for. $U_{c}=[u]_{l_{f}, t_{f}}$. 

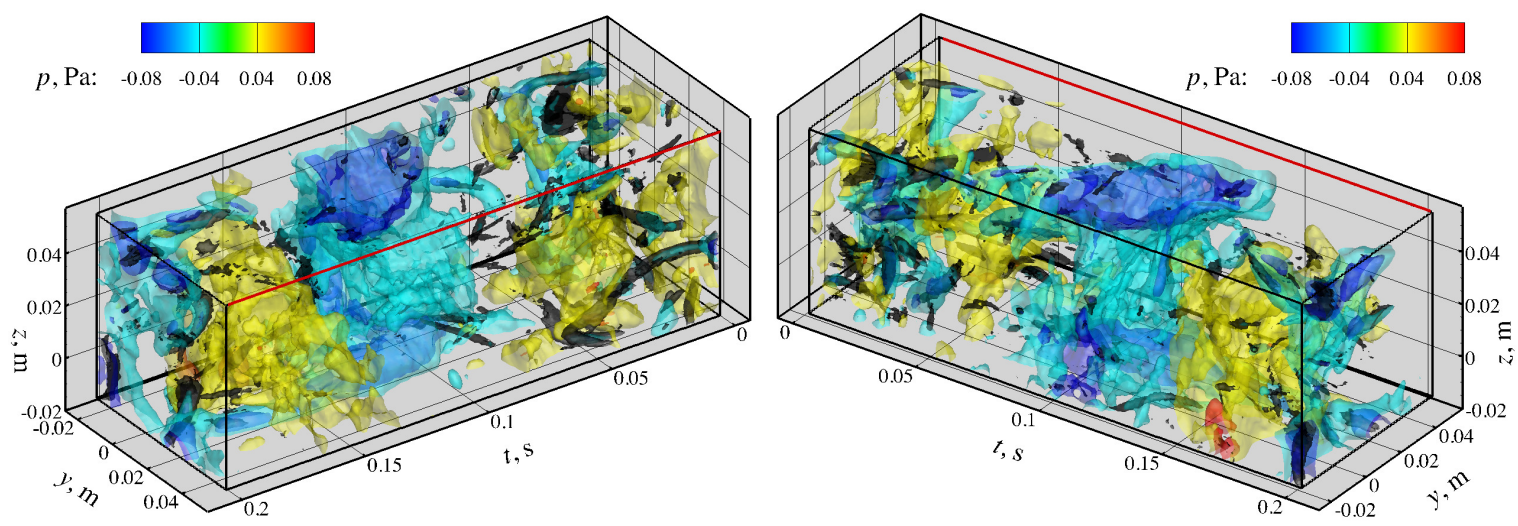

Figure 5: Two views of the space-time volume of pressure for $U_{c}=[u]_{l f}$ with inclusion of viscous terms. The location of the reference pressure is depicted by the red edge. Isocontours of vorticity magnitude $\left(|\omega|=200 \mathrm{~s}^{-1}\right)$ are shown in black. Isosurfaces of pressure are shown for $-0.08 \mathrm{~Pa},-0.04 \mathrm{~Pa}, 0.04 \mathrm{~Pa}$, and $0.08 \mathrm{~Pa}$.

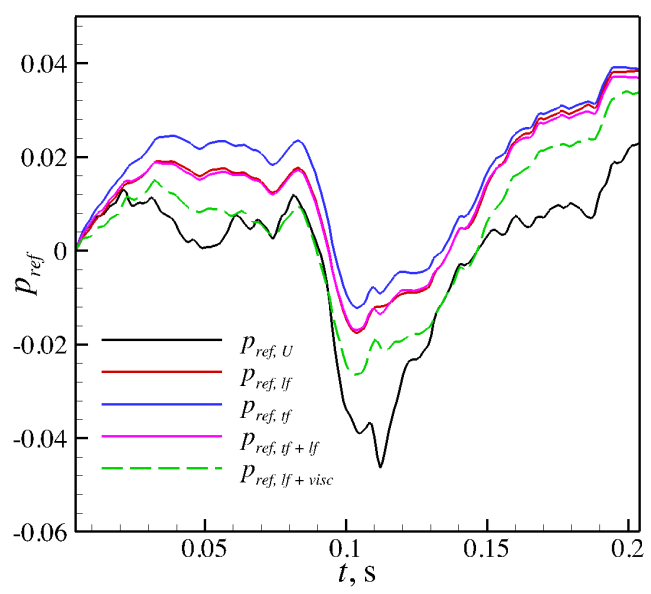

Figure 6: Reference pressures for different convection velocity estimates

assumption. Inspection of the viscous terms, showed that they are an order of magnitude smaller than that of the other terms in the pressure gradient, which suggests they should not play an important role in pressure determination. To assess the influence of excluding viscous terms, we have included them for the for the spatial filtered estimate for convection velocity (3left, blue line). The divergence response is unaffected. Comparing the curl of the in-plane pressure gradient with the results without the viscous terms included (3left, black line), we can see that only for the small filter lengths the result has a slightly different response. From the 'optimal' filter length the results are the same.

Figure 5 shows the resulting space-time pressure volume. Comparing this result with the result for the space filter (4top), we see that at first glance they look very similar. However, if we take a close look, we see that there are subtle changes to the pressure field. The low pressure region in the middle of the volume (light blue) has grown. and the high pressure region at $t=0.17 \mathrm{~s}$ (figure 5 right) has shrunk. This is due to the influence of the viscous term on the reference pressure. Figure 6 shows the reference pressure for all cases, indicating that the differences between the different space-time pressure volumes are primarily caused by these changes in reference pressure. This suggests that the viscous terms are not an important contributor to the pressure.

\subsection{Discussion}

The results show that the mean velocity is not the correct convection velocity for this flow, as evidenced by the oscillations/asymmetries and estimated error on convection velocity. This is not unexpected, since Taylor's hypothesis only holds for flows where the turbulent fluctuation are small. In the jet the turbulent fluctuations are significant $(\approx 30 \%)$. In this situation, there is most likely a range of convection velocities. 
The improvement of the resulting pressure fields when using a filtered instantaneous velocity as convection velocity (even though this still is not the correct convection velocity) suggest that if the convection velocity can be determined within reasonable accuracy, the pressure can be determined using the Taylor's hypothesis pressure determination approach. Space and time filters result in similar pressure fields. However, a filter in space will introduce less divergence than a filter in time.

Regardless, it is not possible to validate the pressure estimates in this flow. The levels of pressure fluctuations $(<0.2 \mathrm{~Pa})$ are too small to be captured with pressure transducers. To our knowledge, the uncertainty of a specialised commercially available pressure transducer after specific calibration is approximately $\pm 0.3 \mathrm{~Pa}$ (e.g. Endevco 8507C-1, see [5]) and will therefore be unable to capture the instantaneous fluctuations observed in this study. Microphones are more sensitive and could be able to capture these small levels of fluctuation. However, the microphones that are able to capture the frequency range and levels of pressure fluctuations accurately are at least $1 / 2$ " in diameter (e.g. Brüel \& Kjaer 4188), which means that they would only be able to capture spatial fluctuations larger than $50 \mathrm{~mm}$. In the current flow, this corresponds to a spatial resolution of about $4 \lambda$ (where $\lambda$ is the Taylor micro scale).

Furthermore, application of a TR-Volume technique for the current problem would result in larger errors than estimated for the TH-TR-Stereo, ranging from $210 \%$ to $40 \%$ (based on the synthetic data analysis for: $W S=2 r_{v}, U_{c}=0.125-1 W S / \Delta t, V_{p} / U_{c}=1$, and $\varepsilon_{u}=4 \%$ ). Applying a Langrangian approach combined with time-resolved volumetric PIV will improve the random noise response at best by a factor of two (optimistic estimate for the improvement based on the region where $U_{c}<1 \mathrm{WS} / \Delta t$ in figure 4(e) from [5]). Apart from difficulties that are associated with implementing this technique (e.g. large volumes needed at high spatial resolution), the improvement of applying a Lagrangian technique would at best make it on par with the error from TH-TR-Stereo in its current implementation.

This suggests that TH-TR-Stereo is the only way to accurately determine pressure fluctuations for turbulent convective flows, provided that an appropriate estimate for the convection velocity can be obtained.

\section{Conclusions}

In this paper, a new approach for pressure estimation in convective flows is proposed. The approach combines volumetric or time-resolved cross-plane measurements with Taylor's hypothesis to calculate pressure.

Based on theoretical estimates and performance assessment on synthetic data it is found that, if the convection velocity is known, this new method is easier to apply and less prone to errors than previous available techniques. The TH-TR-Stereo approach typically out-performs a TR-Volume approach by an order of magnitude in noise response.

Application of the new approach on time-resolved data in the crossflow plane of a turbulent jet shows that, in this case, the mean velocity is the incorrect convection velocity for pressure determination and that a local convection velocity estimate (in-plane filtered axial velocity) reduces the error on the pressure gradient significantly and gives more realistic pressure results. Other than a change in reference pressure for the pressure evaluation, inclusion or omission of the viscous terms appears to not have a significant effect.

All the results appear to indicate that, when applied appropriately, the new approach can be used to determine pressure fluctuations in turbulent convective flows, where, due to their small amplitude and spatial extent, there are no other means to determine pressure fluctuations.

\section{Acknowledgements}

We gratefully acknowledge the support from UK Engineering and Physical Sciences Research Council (EPSRC) through Grant EP/I004785/1 and the European Research Council through the FP7-IDEAS-ERC programme, grant 277472-WBT.

\section{REFERENCES}

[1] Liu X and Katz J "Instantaneous pressure and material acceleration measurements using a four-exposure PIV system" Exp. Fluids 41 (2006) pp. 227-240

[2] Charonko JJ, King CV, Smith BL, and Vlachos PP "Assessment of pressure field calculations from particle image velocimetry measurements" Meas. Sci. Technol. 21 (2010) 105401

[3] Violatio D, Moore P, and Scarano F "Lagrangian and Eulerian pressure field evaluation of rod-airfoil flow from time-resolved tomographic PIV” Exp. Fluids 50(4) (2011) pp. 1057-1070

[4] van Oudheusden "PIV-base pressure measurement" Meas. Sci. Technol. 24 (2013) 032001

[5] de Kat R and van Oudheusden BW “Instantaneous planar pressure determination in turbulent flow” Exp. Fluids 52 (2012) pp. 1089-1106

[6] de Kat R and Ganapathisubramani B "Pressure from particle image velocimetry for convective flows: A Taylors hypothesis approach” Meas. Sci. Technol. 24 (2013) 024002

[7] Ganapathisubramani B, Lakshminarasimhan K, and Clemens NT "Determination of complete velocity gradient tensor by using cinematographic stereoscopic PIV in a turbulent jet" Exp. Fluids 42(6) (2007) pp. 923-939 
[8] Ganapathisubramani B, Lakshminarasimhan K, and Clemens NT "Investigation of three- dimensional structure of fine scales in a turbulent jet by using cinematographic stereoscopic particle image velocimetry" J. Fluid Mech. 598 (2008) pp. 141-175

[9] Elsinga GE, Scarano F, Wieneke B, and van Oudheusden BW “Tomographic particle image velocimetry” Exp. Fluids 41 (2006) pp. 933-947

[10] Elsinga GE, Poelma C, Schröder A, Geisler R, Scarano F, and Westerweel J "Tracking of vortices in a turbulent boundary layer" J. Fluid Mech. 697 (2012) pp. 273-295 